\title{
Body cell mass is a useful parameter for assessing malnutrition and severity of disease in non-ascitic cirrhotic patients with hepatocellular carcinoma or esophageal varices
}

\author{
TAKUMI KAWAGUCHI ${ }^{1,2}$, EITARO TANIGUCHI ${ }^{2}$, MINORU ITOU ${ }^{2}$, RYOKO IBI $^{3}$, TERUYO OKADA ${ }^{3}$, \\ MICHIKO MUTOU ${ }^{3}$, SATOMI SHIRAISHI ${ }^{3}$, YUKI UCHIDA ${ }^{4}$, MOMOKA OTSUKA $^{4}$, \\ YOUKO UMEKI ${ }^{5}$, TETSUHARU ORIISHI ${ }^{2}$, HITOMI HAYABUCHI ${ }^{5}$, \\ SUIKO TANAKA ${ }^{4}$, MACHIKO TAKAKURA ${ }^{3}$ and MICHIO SATA ${ }^{1,2}$ \\ ${ }^{1}$ Department of Digestive Disease Information and Research, ${ }^{2}$ Division of Gastroenterology, Department of Medicine, \\ Kurume University School of Medicine, Kurume; Departments of ${ }^{3}$ Nursing, ${ }^{4}$ Nutrition, Kurume University \\ Hospital, Kurume; ${ }^{5}$ Department of Nutrition and Health Science, Faculty of Human \\ Environmental Science, Fukuoka Women's University, Fukuoka, Japan
}

Received May 26, 2008; Accepted July 14, 2008

DOI: 10.3892/ijmm_00000060

\begin{abstract}
Body cell mass (BCM) is a nutritional parameter, however, changes in BCM in patients with non-ascitic liver cirrhosis (LC) in comparison to patients with other malnutritional diseases remains unclear. We investigated the difference in BCM between patients with LC and malnourished gastrointestinal disease controls (M.CON), and examined the relationship between BCM and the severity of LC. Results demonstrated that serum nutritional parameters were not significantly different between the LC $(n=56)$ and M.CON groups $(\mathrm{n}=25)$, whereas $\mathrm{BCM} \% \mathrm{BW}$ was significantly lower in the LC group than in the M.CON group (50.9 \pm 4.6 vs. $54.4 \pm 7.1 \%, \mathrm{P}=0.018$ ). Furthermore, $\mathrm{BCM} \% \mathrm{BW}$ negatively correlated with the model for end-stage liver disease (MELD) score $(\mathrm{P}=0.04)$. In concluson, $\mathrm{BCM}$ showed a significant decrease and a negative correlation with the MELD score in the LC group. BCM may be a useful parameter for assessing malnutrition and severity of LC.
\end{abstract}

Correspondence to: Dr Takumi Kawaguchi, Department of Digestive Disease Information and Research, Kurume University School of Medicine, 67 Asahi-machi, Kurume 830-0011, Japan E-mail: takumi@med.kurume-u.ac.jp

Abbreviations: BCM, body cell mass; LC, liver cirrhosis; M.CON, malnourished gastrointestinal disease controls; BIA, bioelectrical impedance analyzer; MELD, model for end-stage liver disease; SGA, subjective global assessment; AC, arm circumference; AMC, mid-arm circumference; TSF, tricep skinfold thickness; CV, coefficient of variation; ICF, intracellular fluid; TBW, total body water; ECF, extracellular fluid; FFM, fat free mass

Key words: body composition, eight-polar bioelectrical impedance analyzer, nutritional assessment, liver disease, model for end-stage liver disease score

\section{Introduction}

The liver is one of the central organs involved in the metabolism of various nutrients. In patients with liver cirrhosis, malnutrition is a common feature and is associated with mortality and reduced quality of life (1). Therefore, routine nutritional assessment is recommended in patients with liver cirrhosis $(2,3)$. Subjective global assessment (SGA) and anthropometric measurements are useful techniques for nutritional assessment in various diseases. However, these assessments are not suitable for precise assessment of malnutrition and do not predict a poor clinical outcome in patients with liver cirrhosis (4). Thus, malnutrition is often under-diagnosed in patients with liver cirrhosis.

Significant changes in body composition are known to occur before physiological changes (5). Therefore, measurement of body composition is an integral part of nutritional assessment. Among all body compartments, body cell mass (BCM) has been regarded as the most meaningful for the assessment of malnutrition. BCM comprises the metabolically active and protein-rich compartments in the body and is known to be depleted in case of protein-energy malnutrition (6). Precise estimation of BCM can be obtained by isotope dilution or via the total-body potassium count (7); however, these methods are not generally available for clinical use. Despite certain limitations in patients with ascites, bioelectrical impedance analyzer (BIA) is a useful tool for the estimation of BCM in cirrhotic patients $(8,9)$. An eight-polar BIA has been developed (10) and is currently a non-invasive bedside tool for estimating BCM.

Depletion of BCM is associated with post-liver transplantation mortality, and the measurement of BCM provides clinically relevant information in patients with end-stage liver cirrhosis (11). However, in patients with liver cirrhosis without ascites and/or edema, the utility of BCM as a nutritional parameter remains unclear. In addition, comparisons have been made between cirrhotic patients and healthy 
control subjects, but not malnourished disease controls, in previous studies. In order to verify the specificity for depletion of BCM, cirrhotic patients should be compared to malnourished disease controls. Moreover, the correlation between BCM and severity of disease remains unknown. The aim of this study was to examine changes in BCM in cirrhotic patients in comparison with other malnourished patients and the association between BCM and severity of disease in cirrhotic patients.

\section{Materials and methods}

Subjects. We conducted a cross-sectional study. Via consecutive entry, a total of 56 men with liver cirrhosis (LC) (hepatitis $\mathrm{C}$ virus-related, $\mathrm{n}=36$; hepatitis $\mathrm{B}$ virus-related, $\mathrm{n}=13$; alcoholic, $\mathrm{n}=5$; cryptogenic, $\mathrm{n}=2$ ) and 25 age-matched men with chronic gastrointestinal diseases (inflammatory bowel diseases, $\mathrm{n}=12$; pancreatic cancer, $\mathrm{n}=3$; biliary cancer, $\mathrm{n}=4$; colitis, $\mathrm{n}=3$; colon cancer, $\mathrm{n}=2$; chronic pancreatitis, $\mathrm{n}=1$ ) who served as controls for nutritional status (malnourished disease controls; M.CON) were enrolled in this study during the period from August 2004 to March 2006 at Kurume University Hospital. In the LC group, 28 patients had cooccurring hepatocellular carcinoma. Among them, 20 patients had a single $(<3-\mathrm{cm})$ nodule which had been treated with radiofrequency ablation. Eight patients had advanced tumors which had been treated with chemoembolization. The rest of the 28 patients had co-occurring esophageal varices. Although none of them had episodes of bleeding, they were treated with preventive endoscopic variceal ligation for the esophageal variceal bleeding. The specificity of changes in body composition in cirrhotic patients can be examined by a comparison with malnourished patients. All of the diagnoses were based on clinical, serological, histological, and/or imaging evidence. All patients were hospitalized. For both the LC and M.CON groups, inclusion criteria were: i) Asian men; ii) 20-70 years of age; iii) no edema nor ascites determined by physical examination and ultrasonography; and 4) serum albumin concentration $<4.0 \mathrm{~g} / \mathrm{dl}$. Patients with chronic renal failure or those who had been taking corticosteroids were excluded. The regular hepatic diet (total energy $30-40 \mathrm{kcal} / \mathrm{kg}$ body weight (BW)/day, protein 1.2-1.5 g/ $/ \mathrm{kg} \mathrm{BW/day)} \mathrm{was} \mathrm{fed} \mathrm{to}$ patients in the LC group. The proper diet (total energy 30$35 \mathrm{kcal} / \mathrm{kg} \mathrm{BW} /$ day, protein $1.2-1.5 \mathrm{~g} / \mathrm{kg} \mathrm{BW} /$ day) was fed to patients in the M.CON group. In the analysis for an association between BCM and severity of liver disease, subjects were stratified by age (40-60 years) because of the age-related differences in body water compartments (12). None of the subjects were institutionalized. Informed consent was obtained from each patient. The study protocol conformed to the ethical guidelines of the 1975 Declaration of Helsinki as reflected in a priori approval by the institutional review board.

Laboratory determinations. Venous blood samples were collected in the morning after a 12 -h overnight fast. Hemoglobin concentration, total lymphocyte count, international normalized ratio (INR) of prothrombin time, serum albumin, creatinine and total cholesterol concentration were measured using standard clinical methods (Department of Clinical Laboratory, Kurume University Hospital). A
Table I. Precision of eight-polar BIA in patients with liver cirrhosis.

\begin{tabular}{lccccc}
\hline & Right arm & Left arm & Trunk & Right leg & Left leg \\
\hline $\mathrm{R}_{5}$ & 2.3 & 2.3 & 2.9 & 2.6 & 2.7 \\
$\mathrm{R}_{50}$ & 2.0 & 2.4 & 2.5 & 2.3 & 2.5 \\
$\mathrm{R}_{250}$ & 2.1 & 2.0 & 2.2 & 2.3 & 2.3 \\
$\mathrm{R}_{500}$ & 2.2 & 2.1 & 2.4 & 2.4 & 2.3 \\
\hline
\end{tabular}

Coefficients of variation were calculated from 2 measurements on 7 of the study subjects $(\mathrm{n}=14)$. BIA, direct segmental multifrequency-bioelectrical impedance analyzer; $\mathrm{R}_{\mathrm{x}}$, resistance at $\mathrm{x} \mathrm{kHz}$.

prognostic nutritional index was calculated by using the following formula as previously described (13): Prognostic nutritional index $=10 \mathrm{x}$ Serum albumin concentration $(\mathrm{g} / \mathrm{dl})$ $+0.005 \times$ Total lymphocyte count $\left(\mathrm{mm}^{3}\right)$. Poor prognosis is predicted when the prognostic nutritional index is $<40$ and total lymphocytes count remains $<1000 / \mathrm{mm}^{3}$.

Estimations of anthropometry and body composition. Anthropometry and body composition were estimated by an eight-polar BIA at frequencies of $5,50,250$ and $500 \mathrm{kHz}$ (InBody 3.2, Biospace, Tokyo, Japan). Two electrodes were placed in contact with the palm and thumb of each hand and two electrodes with the anterior and posterior regions of the sole of each foot. Body mass index (BMI) was calculated as $\mathrm{BW}$ in kilograms divided by the square of height in meters $\left(\mathrm{kg} / \mathrm{m}^{2}\right)$. Arm circumference (AC), mid-arm circumference (AMC), tricep skinfold thickness (TSF), waist circumference, hip circumference, and waist-hip ratio were estimated using the software supplied with the eight-polar BIA (Lookin'body 2.0 , Biospace) based on a hydration model (14). The accuracy of the eight-polar BIA analyzer has been previously reported (10). In our preliminary study, anthropometic data estimated by the eight-polar BIA analyzer also showed a significant positive correlation with anthropometic data derived by direct measurement in the LC group (AMC, $n=43$, Spearman rank $\mathrm{r}=0.69, \mathrm{P}<0.0001$; TSF, $\mathrm{n}=43$, Spearman $\operatorname{rank} \mathrm{r}=0.44$, $\mathrm{P}<0.0046)$. AC, AMC and TSF were expressed as a percentage of the expected value for age and gender with reference to Japanese Anthropometric Reference Data (JARD, 2001) (15). The validation of BIA as a measure of change in BCM as estimated by comparing whole-body counting of potassium was reported (16); and therefore, BCM was evaluated by an eight-polar BIA in this study. Because absolute values of body composition vary widely, normalization of body compositions including BCM is required. Height is an index for normalization; however, a balance of each body composition is also important, and normalization of BCM by $\mathrm{BW}$ is used in the definition of HIV-associated malnutrition (17). Therefore, we normalized BCM by BW and the resulting $\mathrm{BCM}$ was expressed as $\mathrm{BCM} \% \mathrm{BW}$ as previously described (11). The precision of InBody 3.2 was determined by measuring resistance two times a day in 7 of the study subjects. The coefficient of variation (CV) [(SD/mean) x 100], calculated from these measurements $(n=14)$ is shown in 
Table II. Nutritional status of the patients.

\begin{tabular}{lccc}
\hline & M.CON & LC & P \\
\hline No. of patients & 25 & 56 & $55.6 \pm 9.1$ \\
Age (years) & $51.9 \pm 13.4$ & $168.1 \pm 6.5$ & N.S. \\
Height $(\mathrm{cm})$ & $165.2 \pm 7.9$ & $7.01 \pm 1.42$ & N.S. \\
Total protein $(\mathrm{g} / \mathrm{dl})$ & $6.71 \pm 0.93$ & $3.24 \pm 0.39$ & N.S. \\
Albumin $(\mathrm{g} / \mathrm{dl})$ & $3.36 \pm 0.43$ & $126 \pm 79$ & N.S. \\
Total cholesterol $(\mathrm{mg} / \mathrm{dl})$ & $157 \pm 90$ & $1280 \pm 704$ & N.S. \\
Total lymphocyte count $\left(/ \mathrm{mm}^{3}\right)$ & $1330 \pm 796$ & $11.9 \pm 1.9$ & N.S. \\
Hemoglobin $(\mathrm{g} / \mathrm{dl})$ & $11.6 \pm 1.9$ & $0.77 \pm 0.17$ & N.S. \\
Creatinine $(\mathrm{mg} / \mathrm{dl})$ & $0.74 \pm 0.26$ & $38.8 \pm 5.0$ & N.S. \\
Prognostic nutritional index & $40.2 \pm 5.6$ & $28 / 56$ & N.S. \\
Accompanying malignancy & $9 / 25$ & N.S. \\
\hline
\end{tabular}

Data are expressed as the mean \pm SD. Prognostic nutritional index was calculated by using the following formula as previously described (13): Prognostic nutritional index $=10 \mathrm{x}$ Serum albumin concentration $(\mathrm{g} / \mathrm{dl})+0.005 \mathrm{x}$ Total lymphocyte count $\left(\mathrm{mm}^{3}\right)$. M.CON, malnourished disease controls; LC, liver cirrhosis.

Table III. Anthropometric assessment.

\begin{tabular}{lccc}
\hline & M.CON & LC & P \\
\hline BMI & $20.8 \pm 3.7$ & $23.1 \pm 3.0$ & 0.009 \\
\%AC & $103.9 \pm 17.9$ & $109.6 \pm 8.9$ & N.S. \\
\%AMC & $100.3 \pm 12.8$ & $107.0 \pm 8.4$ & 0.009 \\
\% TSF & $128.8 \pm 80.9$ & $127.4 \pm 37.9$ & N.S. \\
Waist circumference $(\mathrm{cm})$ & $76.7 \pm 10.2$ & $83.7 \pm 6.6$ & 0.003 \\
Hip circumference $(\mathrm{cm})$ & $88.0 \pm 6.4$ & $92.9 \pm 4.5$ & 0.001 \\
Waist-hip ratio & $0.87 \pm 0.06$ & $0.90 \pm 0.03$ & 0.031 \\
\hline
\end{tabular}

Data are expressed as the mean \pm SD. AC, TSF and AMC are expressed as a percentage of the expected value for age and gender according to the Japanese Anthropometric Reference Data (JARD 2001) (15). M.CON, malnourished disease controls; LC, liver cirrhosis; BMI, body mass index; AC, arm circumference; AMC, arm muscle circumference; TSF, tricep skin-fold thickness.

Table I. Similar to a previous report (10), the CV value was $<3.0$ and comparable to that of a four-polar total-body BIA performed at $50 \mathrm{kHz}(\mathrm{CV}=3.0 \%)(18,19)$.

Severity of liver disease. The severity of cirrhosis was assessed by the model for end-stage liver disease (MELD) score (20). The MELD score was computed using an on-line worksheet (http://www.mayoclinic.org/gi-rst/mayomodel5. html). To avoid negative scores, laboratory values such as serum creatinine concentrations that were $<1 \mathrm{mg} / \mathrm{dl}$ were rounded off to 1 as previously described (21).

Statistical analysis. All data are expressed as the mean \pm SD. Differences between the two groups were analyzed using the Mann-Whitney U test or the Chi-square test. The Spearman's correlation coefficient was calculated for testing the relationship between different quantities in a bivariate regression model using StatView (version 5.1; SAS Institute, Cary, NC). P-values $<0.05$ were indicative of significant differences.

\section{Results}

Patient characteristics. We enrolled 56 patients with liver cirrhosis and 25 patients with chronic gastrointestinal diseases as malnourished disease controls. Clinical and laboratory data for these subjects are summarized in Table II. Age and height were not significantly different between the LC and M.CON groups. Serum concentrations of total protein, albumin, and total cholesterol, total lymphocyte count, hemoglobin concentration, prognostic nutritional index, and the rate accompanying malignancy were also not significantly different between the two groups.

Comparison of anthropometry between the LC and M.CON groups. In the LC group, BMI and \%AMC were significantly higher than in the M.CON group, although \%TSF was not significantly different when comparing the two groups (Table III). Waist and hip circumferences and waist-hip ratio were significantly higher in the LC group than in the M.CON group. 
Table IV. Assessment of body composition.

\begin{tabular}{lccc}
\hline & M.CON & LC & P \\
\hline Fat\%BW & $17.1 \pm 8.4$ & $20.8 \pm 5.1$ & N.S. \\
TBW\%BW & $61.1 \pm 6.4$ & $58.4 \pm 3.8$ & N.S. \\
ECF\%BW & $23.2 \pm 2.8$ & $22.8 \pm 1.6$ & N.S. \\
ICF\%BW & $37.9 \pm 4.8$ & $35.5 \pm 2.3$ & 0.017 \\
ECF/TBW & $0.334 \pm 0.028$ & $0.344 \pm 0.007$ & N.S. \\
Protein\%BW & $16.4 \pm 2.1$ & $15.4 \pm 1.0$ & 0.018 \\
Mineral\%BW & $5.4 \pm 1.0$ & $5.5 \pm 0.4$ & N.S. \\
BCM\%BW & $54.3 \pm 6.9$ & $50.9 \pm 4.5$ & 0.018 \\
\hline
\end{tabular}

Data are expressed as the mean \pm SD. M.CON, malnourished disease controls; LC, liver cirrhosis; BW, body weight; TBW, total body water; ICF, intracellular fluid; ECF, extracellular fluid; BCM, body cell mass.

Comparison of body composition between the LC and M.CON groups. There was a significant decrease in both intracellular fluid (ICF) $\% \mathrm{BW}$ and protein $\% \mathrm{BW}$ in the LC group compared to those in the M.CON group, although fat $\% \mathrm{BW}$, total body water (TBW)\%BW, extracellular fluid (ECF)\%BW, ICF\% TBW, protein \%BW and mineral\%BW did not differ significantly when comparing the two groups (Table IV). Fig. 1A shows the compositions for BW, fat free mass (FFM), soft lean mass (SLM) and BCM. FFM\%BW did not differ between the two groups (Fig. 1B), however, SLM\%BW was significantly decreased in the LC group compared to that in the M.CON group (Fig. 1C). The difference was even more significant when comparing $\mathrm{BCM} \% \mathrm{BW}$ between the two groups (Fig. 1D).

Comparison of nutritional parameters between cirrhotic patients with HCC and cirrhotic patients without HCC. There were no significant differences in nutritional status including biochemical tests between cirrhotic patients with HCC and cirrhotic patients without HCC. No significant differences were also found in anthropometric assessment and body composition between cirrhotic patients with HCC and cirrhotic patients without HCC (data not shown).

An association between BCM and severity of disease in the $L C$ group. Although there was no significant correlation between $\mathrm{BCM}$ and the MELD score when considering all subjects, an association was evident by stratification of the patients in the LC group according to their age. There was a significant negative correlation between BCM and the MELD score in cirrhotic patients aged $40-60$ years $\left(n=29, R^{2}=0.148, P=0.04\right)$ (Fig. 2).

\section{Discussion}

In this study, we demonstrated a significant decrease in BCM in patients with liver cirrhosis compared to that in malnourished patients with chronic gastrointestinal diseases, although serum or hematological nutritional parameters were not significantly different between the two groups. Moreover,
A

Body Weight (BW)
Fat Free Mass (FFM)
Soft Lean Mass (SLM)
Body Cell Mass (BCM)

Fat Mineral Protein ICF ECF

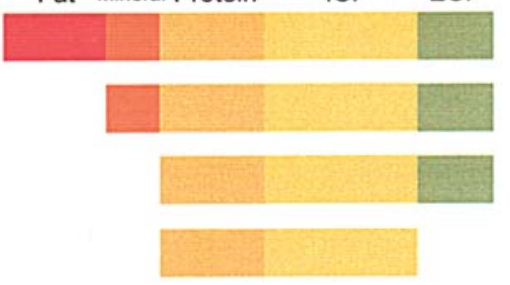

B

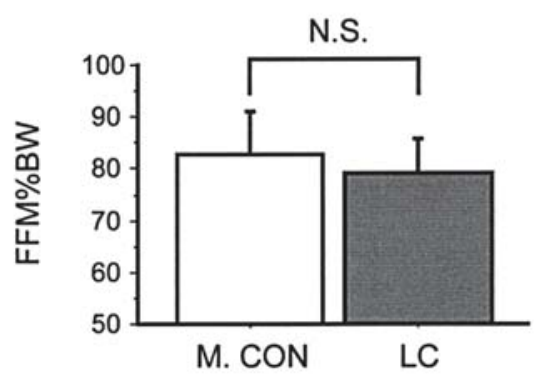

C



D

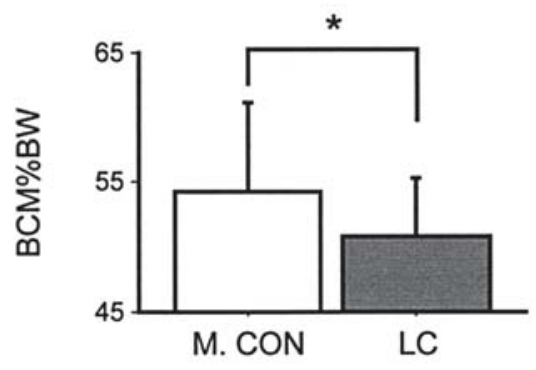

Figure 1. Scheme of body composition (A). Comparison of FFM (B), SLM (C) and BCM (D) between the LC and M.CON groups. Values are expressed as the mean \pm SD. Differences between the two groups were analyzed using the Mann-Whitney U test. N.S., not significant. ${ }^{*} \mathrm{P}<0.05$. BW, body weight; ICF, intracellular fluid; ECF, extracellular fluid; M.CON, malnourished disease controls; LC, liver cirrhosis.

we showed a significant negative correlation between BCM and the MELD score in patients with liver cirrhosis.

Significant changes in body composition are known to occur before physiological changes (5). Similar to previous reports $(22,23)$, an increase in TBW and ECW was also noted in this study when these body compositions were normalized with height (data not shown). In addition, by normalization with $\mathrm{BW}$, we found that $\mathrm{ICF} \% \mathrm{BW}$ and protein $\% \mathrm{BW}$ were significantly decreased in the LC group compared to those in the M.CON group. In agreement with our data, Figueiredo et al recently reported that reduction in ICF, but not fat, occurred 


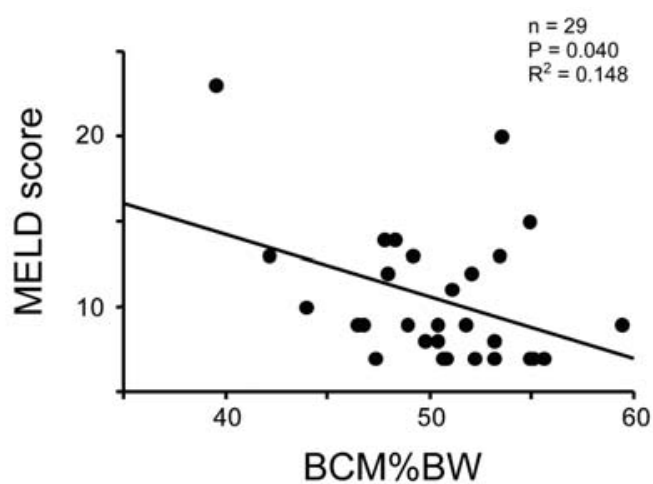

Figure 2. An association between BCM and severity of disease in patients with liver cirrhosis. Subjects was stratified by age because of the age difference in body water compartments (12) (See Materials and methods). An association between BCM and MELD score was analyzed by using the Spearman's correlation coefficient in a bivariate regression model. $\mathrm{P}<0.05$, significant difference. MELD, the model for end-stage liver disease; BCM, body cell mass; BW, body weight.

even before fluid retention was noted clinically (22). We did not investigate the mechanisms for decreases in ICF and protein. However, one would think that loss of body protein leads to a decrease in intracellular osmotic pressure and subsequent decrease in ICF, since the rate of endogenous protein degradation is increased in patients with liver cirrhosis (24).

In this study, both groups manifested a characteristic of protein-energy malnutrition. Although we do not have a normal value for BCM for healthy males in Japan, it is believed to be approximately $60 \%$ of $\mathrm{BW}$ according to the average value for body compartments $(20 \%$ of ICF\%BW plus $40 \%$ of protein $\% \mathrm{BW}$ ). In the M.CON group, BCM\%BW was $<60 \%$ of $\mathrm{BW}$ which is considered to be lower than that of normal subjects. In the LC group, BCM\%BW was also $<60 \%$ of BW. In addition, BCM was significantly decreased in the LC group compared to the M.CON group, although serum and hematologic nutritional parameters were not significantly different. These findings may indicate that change in body composition is more evident in the LC group than in the M.CON group.

There were no significant differences in nutritional parameters between cirrhotic patients with HCC and cirrhotic patients without HCC. Occurrence of HCC seems to affect the metabolic course of chronic liver disease; however, our data did not show any difference. One would think that HCC does not affect nutritional parameters, because $>70 \%$ of cirrhotic patients with HCC showed a single $(<3-\mathrm{cm})$ nodule treated with curative therapy. Alternatively, cirrhotic patients without HCC were admitted for preventive therapy of esophageal varices. Thus, cirrhotic patients without HCC also had the complication of LC and therefore, no significant differences in nutritional parameters could be found.

We also showed the significance of BCM in cirrhotic patients. A significant negative correlation between BCM and the MELD score was observed in cirrhotic patients without ascites and/or edema. On the other hand, Muller et al reported that there was no significant difference in BCM among patients according to the Child-Pugh classification (25).
Although the reason for this discrepancy is unclear, the explanation may be that BCM was evaluated as the absolute value in the previous study. BW increases with increasing severity in liver disease. Therefore, normalization of BCM by BW could be one of the reasons for this discrepancy. In accordance with our data, Figueiredo et al reported that normalized BCM is correlated with the Child-Pugh classification (22). Thus, the estimation of BCM is not only non-invasive but also meaningful. However, we have to be cautious in interpreting BCM values when patients exhibit hyperbilirubinemia. In our analysis for an association between BCM and the MELD score, some patient data did not correspond with the regression line, and hyperbilirubinemia (>6 mg/dl of serum bilirubin levels) was a common characteristic in these patients. The relationship between hyperbilirubinemia and impedance is unclear. However, bilirubin is known to modulate cell-cell contact and results in changes in transepithelial electrical resistance (26). Thus, BCM estimated by eight-polar BIA may not predict prognosis in patients with hyperbilirubinemia.

The limitations of this study included the small sample size, the ratio of cases to controls, and the heterogeneity of the malnourished controls. In order to confirm the significance of BCM in patients with LC, a large-scale multicenter clinical study including healthy individuals is required. In addition, the use of the MELD score may not have been suitable for this study. Since the significance of the correlation between BCM and MELD was poor, the real prognostic relevance of $\mathrm{BCM}$ should be evaluated in future studies. Moreover, the number of hospitals that are able to measure BCM is still limited. Changes in BCM are known to correlate with energy and protein intake (27). Thus, food consumption should be assessed cautiously in order to prevent underestimations that may occur by using traditional nutritional assessment measures.

In conclusion, we demonstrated a significant decrease in $\mathrm{BCM}$ in patients with liver cirrhosis compared to that in malnourished patients with chronic gastrointestinal diseases. We also showed a significant negative correlation between BCM and MELD score in cirrhotic patients without ascites and/or edema. Thus, BCM may be a useful parameter for assessing malnutrition and severity of disease in patients with liver cirrhosis.

\section{Acknowledgements}

This study was supported, in part, by a Grant-in-Aid for Young Scientists (B) (no. 19790643 to T.K.).

\section{References}

1. Alberino F, Gatta A, Amodio P, et al: Nutrition and survival in patients with liver cirrhosis. Nutrition 17: 445-450, 2001.

2. Morgan MY, Madden AM, Soulsby CT and Morris RW: Derivation and validation of a new global method for assessing nutritional status in patients with cirrhosis. Hepatology 44: 823-835, 2006.

3. Plauth M, Cabre E, Riggio O, et al: ESPEN Guidelines on Enteral Nutrition: Liver disease. Clin Nutr 25: 285-294, 2006.

4. Alvares-da-Silva MR and Reverbel da Silveira T: Comparison between handgrip strength, subjective global assessment, and prognostic nutritional index in assessing malnutrition and predicting clinical outcome in cirrhotic outpatients. Nutrition 21: 113-117, 2005. 
5. Crawford DH, Shepherd RW, Halliday JW, et al: Body composition in nonalcoholic cirrhosis: the effect of disease etiology and severity on nutritional compartments. Gastroenterology 106: 1611-1617, 1994.

6. Pirlich M, Schutz T, Spachos T, et al: Bioelectrical impedance analysis is a useful bedside technique to assess malnutrition in cirrhotic patients with and without ascites. Hepatology 32: 1208-1215, 2000

7. Morgan MY and Madden AM: The assessment of body composition in patients with cirrhosis. Eur J Nucl Med 23: 213-225, 1996.

8. Henkel AS and Buchman AL: Nutritional support in patients with chronic liver disease. Nat Clin Pract Gastroenterol Hepatol 3: 202-209, 2006.

9. Pirlich M, Schutz T, Ockenga J, et al: Improved assessment of body cell mass by segmental bioimpedance analysis in malnourished subjects and acromegaly. Clin Nutr 22: 167-174, 2003.

10. Bedogni G, Malavolti M, Severi S, et al: Accuracy of an eightpoint tactile-electrode impedance method in the assessment of total body water. Eur J Clin Nutr 56: 1143-1148, 2002.

11. Selberg O, Bottcher J, Tusch G, Pichlmayr R, Henkel E and Muller MJ: Identification of high- and low-risk patients before liver transplantation: a prospective cohort study of nutritional and metabolic parameters in 150 patients. Hepatology 25: 652-657, 1997

12. Lesser GT and Markofsky J: Body water compartments with human aging using fat-free mass as the reference standard. Am $\mathbf{J}$ Physiol 236: R215-R220, 1979.

13. Buzby GP, Mullen JL, Matthews DC, Hobbs CL and Rosato EF: Prognostic nutritional index in gastrointestinal surgery. Am J Surg 139: 160-167, 1980.

14. Wang Z, Deurenberg P, Wang W, Pietrobelli A, Baumgartner RN and Heymsfield SB: Hydration of fat-free body mass: new physiological modeling approach. Am J Physiol 276: E995-E1003, 1999.

15. Okada T, Muto Y, Yamamori H, et al: Japanese Anthropometric Reference Data; JARD 2001. Jpn J Nutr Assess 19: 1-82, 2002.

16. Kotler DP, Rosenbaum K, Allison DB, Wang J and Pierson RN Jr: Validation of bioimpedance analysis as a measure of change in body cell mass as estimated by whole-body counting of potassium in adults. JPEN J Parenter Enteral Nutr 23: 345-349, 1999.
17. Polsky B, Kotler D and Steinhart C: HIV-associated wasting in the HAART era: guidelines for assessment, diagnosis, and treatment. AIDS Patient Care STDS 15: 411-423, 2001.

18. Heitmann BL: Impedance: a valid method in assessment of body composition? Eur J Clin Nutr 48: 228-240, 1994.

19. Kushner RF and Schoeller DA: Estimation of total body water by bioelectrical impedance analysis. Am J Clin Nutr 44: 417-424, 1986.

20. Kamath PS, Wiesner RH, Malinchoc M, et al: A model to predict survival in patients with end-stage liver disease. Hepatology 33: 464-470, 2001.

21. Wiesner R, Edwards E, Freeman R, et al: Model for end-stage liver disease (MELD) and allocation of donor livers. Gastroenterology 124: 91-96, 2003.

22. Figueiredo FA, De Mello Perez R and Kondo M: Effect of liver cirrhosis on body composition: evidence of significant depletion even in mild disease. J Gastroenterol Hepatol 20: 209-216, 2005.

23. McCullough AJ, Mullen KD and Kalhan SC: Measurements of total body and extracellular water in cirrhotic patients with and without ascites. Hepatology 14: 1102-1111, 1991.

24. Dichi JB, Dichi I, Maio R, et al: Whole-body protein turnover in malnourished patients with child class B and C cirrhosis on diets low to high in protein energy. Nutrition 17: 239-242, 2001.

25. Muller MJ, Lautz HU, Plogmann B, Burger M, Korber J and Schmidt FW: Energy expenditure and substrate oxidation in patients with cirrhosis: the impact of cause, clinical staging and nutritional state. Hepatology 15: 782-794, 1992.

26. Raimondi F, Crivaro V, Capasso L, et al: Unconjugated bilirubin modulates the intestinal epithelial barrier function in a human-derived in vitro model. Pediatr Res 60: 30-33, 2006.

27. Robert S, Zarowitz BJ, Hyzy R, Eichenhorn M, Peterson EL and Popovich J Jr: Bioelectrical impedance assessment of nutritional status in critically ill patients. Am J Clin Nutr 57: 840-844, 1993. 